\title{
An Alternate Set of Basis Functions for the Electromagnetic Solution of Arbitrarily-Shaped, Three-Dimensional, Closed, Conducting Bodies Using Method of Moments
}

\author{
Anne I. Mackenzie \\ NASA Langley Research Center, \\ Hampton, VA 23681 (USA) \\ email:anne.mackenzie-1@nasa.gov \\ Michael E. Baginski \\ Department of E \& CE, \\ Auburn University, Auburn, AL 36849 (USA) \\ email:baginme@auburn.edu \\ Sadasiva M. Rao \\ Department of E \& CE, \\ Auburn University, Auburn, AL 36849 (USA) \\ email:rao@eng.auburn.edu
}

\begin{abstract}
In this work, we present an alternate set of basis functions, each defined over a pair of planar triangular patches, for the method of moments solution of electromagnetic scattering and radiation problems associated with arbitrarily-shaped, closed, conducting surfaces. The present basis functions are point-wise orthogonal to the pulse basis functions previously defined. The prime motivation to develop the present set of basis functions is to utilize them for the electromagnetic solution of dielectric bodies using a surface integral equation formulation which involves both electric and magnetic currents. However, in the present work, only the conducting body solution is presented and compared with other data.
\end{abstract}




\section{Introduction}

In a recent paper, we have introduced a new set of pulse-type basis functions, each defined over a pair of planar triangular patches, to calculate the electromagnetic scattering/radiation associated with three-dimensional, arbitrarilyshaped conducting bodies [1]. The primary motivation for this work is to develop an efficient and well-conditioned method of moments (MoM) [2] solution for dielectric material bodies via a surface integral equation (SIE) approach [3]. It may be noted that since the SIE approach involves both electric and magnetic currents as unknowns in the MoM formulation, it is necessary to define two mutually orthogonal sets of basis functions to generate a wellconditioned moment matrix. In our previous paper, we have presented one set of basis functions and their suitability to calculate electromagnetic scattering from arbitrarily-shaped conducting bodies. In this work, we present the alternate set of basis functions and the numerical solution scheme to solve the same class of problems. We emphasize here that the mathematical equations appearing in the dielectric body SIE formulation are very similar to the equations we encounter in the PEC body problem, and, therefore, solving the PEC case provides confidence in the solution methodology.

\section{Description of the Problem}

Let $S$ denote the surface of an arbitrarily-shaped, perfectly conducting, closed body illuminated by an incident electromagnetic plane wave $\boldsymbol{E}^{i}$ as shown in Figure 1. Using the equivalence principle, potential theory, and the freespace Green's function [4], the electric field integral equation (EFIE) is given by

$$
[j \omega \boldsymbol{A}+\nabla \Phi]_{t a n}=\boldsymbol{E}_{\text {tan }}^{i}
$$

where the subscript "tan" refers to the tangential component. In (1),

$$
\begin{aligned}
\boldsymbol{A} & =\mu \int_{S} \boldsymbol{J}_{S} G d S^{\prime} \\
\Phi & =\epsilon^{-1} \int_{S} q_{S} G d S^{\prime} \\
G & =\frac{e^{-j k R}}{4 \pi R} \\
R & =\left|\boldsymbol{r}-\boldsymbol{r}^{\prime}\right|
\end{aligned}
$$


$\epsilon$ and $\mu$ are the permittivity and permeability of the surrounding medium, $k$ is the wave number and $\boldsymbol{r}$ and $\boldsymbol{r}^{\prime}$ represent the position vectors to observation and source points, respectively, from a global coordinate origin. The unknown surface current $\boldsymbol{J}_{S}$ is related to the charge density $q_{S}$ by the continuity equation, given by

$$
\nabla \bullet \boldsymbol{J}_{S}=-j \omega q_{S}
$$

For the numerical solution of (1), we apply the method of moments formulation using planar triangular patch modeling and the basis functions as described in the following:

\section{Description of Basis Functions}

Let $T_{n}^{+}$and $T_{n}^{-}$represent two triangles connected to the edge $n$ of the triangulated surface model as shown in Figure 2. We define two mutually orthogonal vector basis functions associated with the $n^{\text {th }}$ edge as

$$
\begin{aligned}
\boldsymbol{f}_{n}(\boldsymbol{r}) & = \begin{cases}\hat{\boldsymbol{n}}^{ \pm} \times \hat{\boldsymbol{\ell}}, \quad \boldsymbol{r} \in S_{n}, \\
0, & \text { otherwise }\end{cases} \\
\text { and } & \\
\boldsymbol{g}_{n}(\boldsymbol{r}) & = \begin{cases}\hat{\boldsymbol{\ell}}, & \boldsymbol{r} \in S_{n}, \\
0, & \text { otherwise }\end{cases}
\end{aligned}
$$

where $S_{n}$ represents the region obtained by connecting the mid-points of the free edges to the centroids of triangles $T_{n}^{ \pm}$and to the nodes of edge $n$. This area is shown shaded in Figure 2. Also, $\hat{\boldsymbol{\ell}}$ and $\hat{\boldsymbol{n}}^{ \pm}$represent the unit vector along the $n^{\text {th }}$ edge and the unit vector normal to the plane of the triangle $T_{n}^{ \pm}$, respectively. Note that the basis functions defined in $(7,8)$ are actually pulse functions, point-wise orthogonal to each other, defined over the region $S_{n}$. The electromagnetic solution procedure using the basis functions $\boldsymbol{f}_{n}$ is described in [1], and in this work we present a similar solution using the basis functions $\boldsymbol{g}_{n}$.

It may also be noted here that the basis functions $\boldsymbol{g}_{n}$ are less versatile than the basis functions $\boldsymbol{f}_{n}$. Unlike the basis functions $\boldsymbol{f}_{n}$, which are applicable to both open and closed bodies as demonstrated in [1], the basis functions $\boldsymbol{g}_{n}$ are applicable to closed bodies only. The main reason for this restriction is that the functions $\boldsymbol{g}_{n}$ are defined parallel to the edges of the planar triangular 
patches and represent the tangential component of the current. It is wellknown that the tangential component of the surface current is undefined for an open surface at the boundary. However, our primary motivation to use these functions is their applicability in the solution of the dielectric body problem, which is always posed for a closed body. Hence, the basis functions $\boldsymbol{g}_{n}$ do not hinder our purpose.

\section{Numerical Solution Procedure}

As a first step, we consider the testing procedure. Consider the $m^{\text {th }}$ interior edge, associated with triangles $T_{m}^{ \pm}$. We integrate the vector component of (1) along the $m^{\text {th }}$ edge to obtain

$$
\int_{\boldsymbol{\ell}_{m}} j \omega \boldsymbol{A}(\boldsymbol{r}) \bullet d \boldsymbol{\ell}+\int_{\boldsymbol{\ell}_{m}} \nabla \Phi(\boldsymbol{r}) \bullet d \boldsymbol{\ell}=\int_{\boldsymbol{\ell}_{m}} \boldsymbol{E}^{i}(\boldsymbol{r}) \bullet d \boldsymbol{\ell}
$$

which may be re-written as

$$
\int_{\boldsymbol{\ell}_{m}} j \omega \boldsymbol{A}(\boldsymbol{r}) \bullet d \boldsymbol{\ell}+\Phi_{n}^{m 2}-\Phi_{n}^{m 1}=\int_{\boldsymbol{\ell}_{m}} \boldsymbol{E}^{i}(\boldsymbol{r}) \bullet d \boldsymbol{\ell}
$$

for $n=1,2,3, \cdots, N$, where $n$ represents the source charge region and $N$ represents the total number of edges in the triangulation scheme. Note that in (10), $\Phi_{n}^{m 1}$ and $\Phi_{n}^{m 2}$ represent the scalar potentials evaluated at the nodes connected to the $m^{\text {th }}$ edge. Further, the integrals appearing in (10) may be easily evaluated using any accurate numerical integration algorithm such as the 1-point, 2-point or 4-point trapezoidal rule. In this work, we choose to use a 2-point method.

Next, we consider the expansion procedure. Using the basis functions $\boldsymbol{g}_{n}$ defined in (8), we approximate the unknown current $\boldsymbol{J}$ as

$$
\boldsymbol{J}=\sum_{n=1}^{N} I_{n} \boldsymbol{g}_{n} .
$$

This is followed by a substitution of the current expansion (11) into (10), yielding a $N \times N$ system of linear equations which may be written in matrix form as $\boldsymbol{Z} \boldsymbol{I}=\boldsymbol{V}$, where $\boldsymbol{Z}=\left[Z_{m n}\right]$ is an $N \times N$ matrix and $\boldsymbol{I}=\left[I_{n}\right]$ and $\boldsymbol{V}=\left[V_{m}\right]$ are column vectors of length $N$. The elements of the $\boldsymbol{Z}$ and $\boldsymbol{V}$ are 
given by

$$
\begin{aligned}
Z_{m n} & =j \omega \int_{\boldsymbol{\ell}_{m}} \boldsymbol{A}_{n} \bullet d \boldsymbol{\ell}+\Phi_{n}^{m 2}-\Phi_{n}^{m 1} \\
V_{m} & =\int_{\boldsymbol{\ell}_{m}} \boldsymbol{E}^{i} \bullet d \boldsymbol{\ell}
\end{aligned}
$$

where

$$
\begin{aligned}
\boldsymbol{A}_{n} & =\mu \int_{S} \boldsymbol{g}_{n} \frac{e^{-j k R}}{4 \pi R} d S^{\prime} \\
\Phi_{n}^{m 1} & =\frac{-1}{j \omega \epsilon} \int_{S} \nabla_{s} \bullet \boldsymbol{g}_{n} \frac{e^{-j k R_{n}^{m 1}}}{4 \pi R_{n}^{m 1}} d S^{\prime} \\
\Phi_{n}^{m 2} & =\frac{-1}{j \omega \epsilon} \int_{S} \nabla_{s} \bullet \boldsymbol{g}_{n} \frac{e^{-j k R_{n}^{m 2}}}{4 \pi R_{n}^{m 2}} d S^{\prime} \\
R & =\left|\boldsymbol{r}-\boldsymbol{r}^{\prime}\right| \\
R_{n}^{m 1} & =\left|\boldsymbol{r}_{m 1}-\boldsymbol{r}^{\prime}\right| \\
R_{n}^{m 2} & =\left|\boldsymbol{r}_{m 2}-\boldsymbol{r}^{\prime}\right|
\end{aligned}
$$

and $\boldsymbol{r}_{m 1}$ and $\boldsymbol{r}_{m 2}$ are the position vectors to the nodes $m 1$ and $m 2$, respectively, connected to the $m^{\text {th }}$ edge.

The numerical evaluation of the vector potential, shown in (14), is straightforward and may be accomplished by the procedure described in [5]. However, the numerical evaluation of the scalar potential terms described in (15) and (16) may be carried out as follows:

Let us define the unknown charge density $q_{S}$ in (3) as

$$
q_{S}=\sum_{i=1}^{N_{n}} \alpha_{i} P_{i}
$$

where $N_{n}$ represents the total number of nodes (vertices) in the model and

$$
P_{i}(\boldsymbol{r})= \begin{cases}1, & \boldsymbol{r} \in S_{i} \\ 0, & \text { otherwise }\end{cases}
$$

In (21), $S_{i}$ is the $i^{\text {th }}$ charge patch, formed by connecting the centers of the edges and the centroids of the triangles associated with the node i, as shown by the shaded area in Figure 3. 
Now, let us consider the $i^{\text {th }}$ charge patch. Using (6), the well-known Divergence theorem and simple vector calculus, we have

$$
\begin{aligned}
\int_{S_{i}} q_{S} d S & =\int_{S_{i}} \frac{\nabla_{s} \bullet \boldsymbol{J}}{-j \omega} d S \\
& =\frac{j}{\omega} \oint_{C_{i}} \boldsymbol{J} \bullet \hat{\boldsymbol{n}}_{\ell} d \ell
\end{aligned}
$$

where $C_{i}$ is the contour bounding the charge patch $S_{i}$, and $\hat{\boldsymbol{n}}_{\ell}$ is the unit vector normal to the contour $C_{i}$ in the plane of the triangle containing the contour segment. By considering the left hand side of (22), we have

$$
\begin{aligned}
\int_{S_{i}} q_{S} d S & =\int_{S_{i}} \alpha_{i} d S \\
& =\alpha_{i} A_{S i}
\end{aligned}
$$

where $A_{S i}$ represents the area of the charge patch $S_{i}$. Considering the right hand side of (22), we have

$$
\frac{j}{\omega} \oint_{C_{i}} \boldsymbol{J} \bullet \hat{\boldsymbol{n}}_{\ell} d \ell=\frac{j}{\omega} \sum_{j=1}^{E_{K}} I_{i j}\left[\hat{\boldsymbol{\ell}}_{i j} \bullet\left(\boldsymbol{r}_{i j}^{c+} \times \hat{\boldsymbol{n}}_{i j}^{+}+\left(\boldsymbol{r}_{i j}^{c-} \times \hat{\boldsymbol{n}}_{i j}^{-}\right)\right]\right.
$$

where $\hat{\boldsymbol{\ell}}_{i j}, \boldsymbol{r}_{i j}^{c \pm}$, and $\hat{\boldsymbol{n}}_{i j}^{ \pm}$represent the unit vector along the $j^{\text {th }}$ edge connected to node $i$, the vector from the center of the $j^{\text {th }}$ edge to the centroid of the \pm triangle connected to the edge, and the unit vector normal to the plane of the \pm triangle associated with the $j^{\text {th }}$ edge connected to the node $i$, respectively. Also, $E_{K}$ represents the total number of edges connected to node $i$. Using (23) and (24), we have

$$
\alpha_{i}=\frac{j}{\omega A_{S i}} \sum_{j=1}^{E_{K}} I_{i j}\left[\hat{\ell}_{i j} \bullet\left(\boldsymbol{r}_{i j}^{c+} \times \hat{\boldsymbol{n}}_{i j}^{+}+\left(\boldsymbol{r}_{i j}^{c-} \times \hat{\boldsymbol{n}}_{i j}^{-}\right)\right] .\right.
$$

Thus, we can write the scalar potential terms in (15) and (16) as

$$
\begin{aligned}
\Phi_{n}^{m 1} & =\frac{j}{\omega \epsilon}\left[\frac{\hat{\boldsymbol{\ell}}_{n} \bullet\left(\boldsymbol{r}_{n}^{c+} \times \hat{\boldsymbol{n}}_{n}^{+}\right)}{A_{S n 1}} \int_{S_{n 1}} \frac{e^{-j k R_{n 1}^{m 1}}}{4 \pi R_{n 1}^{m 1}} d S^{\prime}\right. \\
& \left.+\frac{\hat{\boldsymbol{\ell}}_{n} \bullet\left(\boldsymbol{r}_{n}^{c-} \times \hat{\boldsymbol{n}}_{n}^{-}\right)}{A_{S n 2}} \int_{S_{n 2}} \frac{e^{-j k R_{n 2}^{m 1}}}{4 \pi R_{n 2}^{m 1}} d S^{\prime}\right]
\end{aligned}
$$




$$
\begin{aligned}
\Phi_{n}^{m 2} & =\frac{j}{\omega \epsilon}\left[\frac{\hat{\boldsymbol{\ell}}_{n} \bullet\left(\boldsymbol{r}_{n}^{c+} \times \hat{\boldsymbol{n}}_{n}^{+}\right)}{A_{S n 1}} \int_{S_{n 1}} \frac{e^{-j k R_{n 1}^{m 2}}}{4 \pi R_{n 1}^{m 2}} d S^{\prime}\right. \\
& \left.+\frac{\hat{\boldsymbol{\ell}}_{n} \bullet\left(\boldsymbol{r}_{n}^{c-} \times \hat{\boldsymbol{n}}_{n}^{-}\right)}{A_{S n 2}} \int_{S_{n 2}} \frac{e^{-j k R_{n 2}^{m 2}}}{4 \pi R_{n 2}^{m 2}} d S^{\prime}\right] \\
R_{n j}^{m i} & =\left|\boldsymbol{r}_{m i}-\boldsymbol{r}_{n j}\right| \quad i=1,2 \text { and } j=1,2
\end{aligned}
$$

where $\boldsymbol{r}_{m 1}, \boldsymbol{r}_{m 2}, \boldsymbol{r}_{n 1}$, and $\boldsymbol{r}_{n 2}$ are the position vectors to nodes $m 1$ and $m 2$ connected to the edge $m$, and nodes $n 1$ and $n 2$ connected to the edge $n$, respectively. Further, $S_{n 1}$ and $S_{n 2}$ are the charge patches associated with the nodes $n 1$ and $n 2$, respectively, as depicted in Figure 3 . The integrals in (26) and (27) may be evaluated with the procedures described in [5].

Finally, once the matrices $\boldsymbol{Z}$ and $\boldsymbol{V}$ are determined, we may easily solve the system of linear equations to obtain $\boldsymbol{I}$.

\section{$5 \quad$ Numerical Results}

In this section, we present numerical results for a PEC sphere (diameter $=0.15 \lambda$ ) and a cube (length $=0.15 \lambda)$, and we compare the results with the solution obtained using the procedure presented in [1]. Also, for the case of the sphere, the results are compared with the exact solution. The sphere and the cube are modeled with 500 and 960 triangles, respectively. For both examples, the body is placed symmetrically at the center of the coordinate system and illuminated by an x-polarized plane wave traveling along the z-axis. The bistatic radar cross section is presented in Figures 4 and 5. We note that the new basis function results compare well with the other methods.

\section{Conclusions}

In this work, we present a new set of basis functions for the method of moments solution of electromagnetic scattering by conducting bodies of arbitrary shape. The new basis functions are pulse basis functions defined over a pair of triangular patches and tangential to the common edge. The present set of basis functions along with the functions defined in our previous work [1] should prove helpful in obtaining a stable and well-conditioned solution to the material body problem. At present, the work is in progress to apply 
these two sets of basis functions to material bodies, and the results will be reported in the near future.

\section{References}

[1] A. I. Mackenzie, M. E. Baginski, and S. M. Rao, "New basis functions for the electromagnetic solution of arbitrarily-shaped, three-dimensional conducting bodies using method of moments," Accepted for publication in Microwave and Optical Technology Letters.

[2] R. F. Harrington, Field Computation by Moment Methods, New York, Macmillan, 1968.

[3] A. J. Poggio and E. K. Miller, "Integral Equation Solution of Three Dimensional Scattering Problems," in Computer Techniques for Electromagnetics, R. Mittra, Ed., England: Pergammon, 1973, Ch 4.

[4] R. F. Harrington, Time-Harmonic Electromagnetic Fields, New York, McGraw-Hill, 1961.

[5] D. R. Wilton, S. M. Rao, A. W. Glisson, D. H. Schaubert, O. M. Al-Bundak, and C. M. Butler, "Potential Integrals for Uniform and Linear Source Distributions on Polygonal and Polyhedral Domains," IEEE Transactions on Antennas and Propagation, vol. 32, pp. 276 - 281, March 1984. 


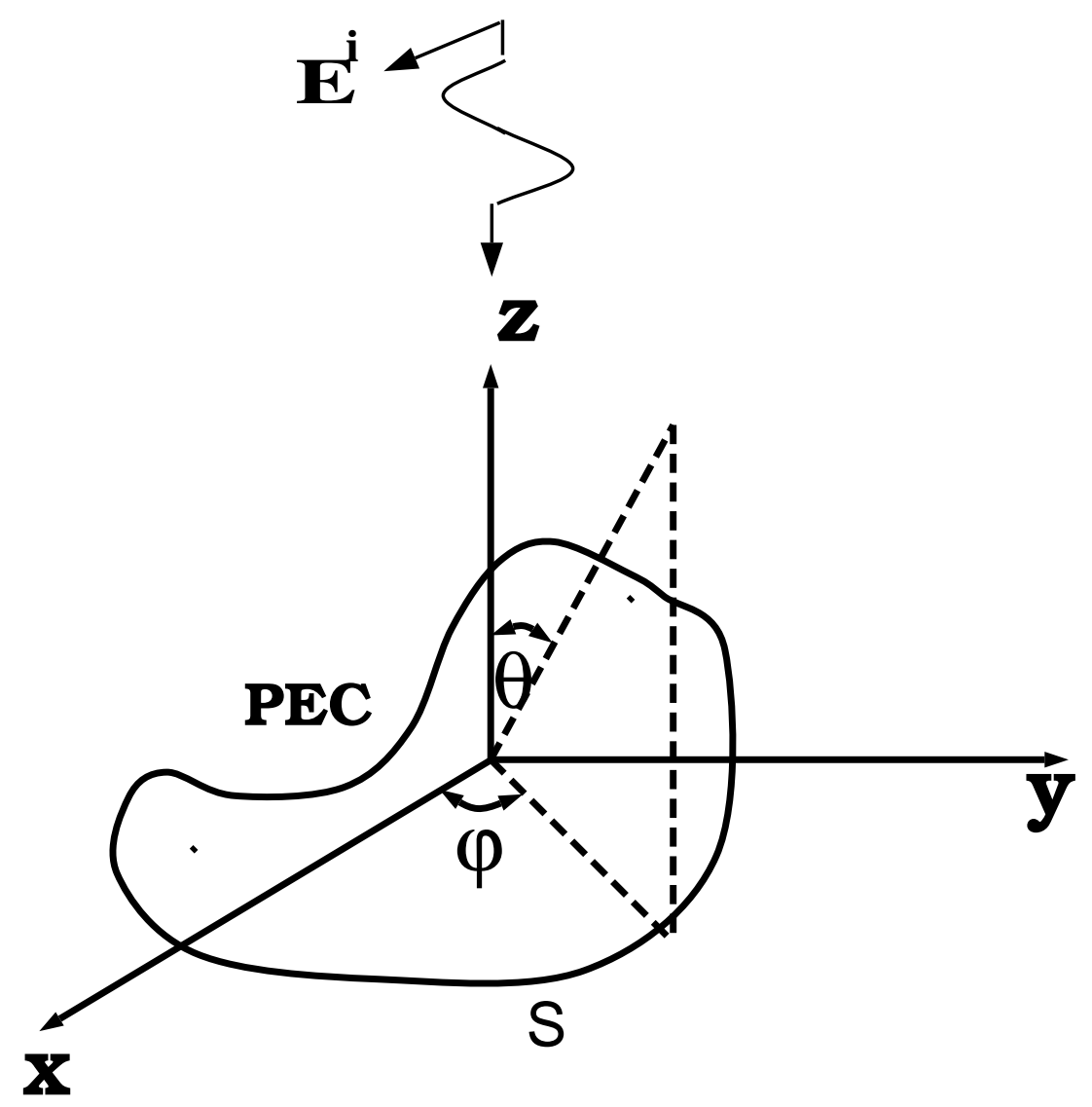

Figure 1: Arbitrarily-shaped conducting body excited by an incident electromagnetic plane wave. 


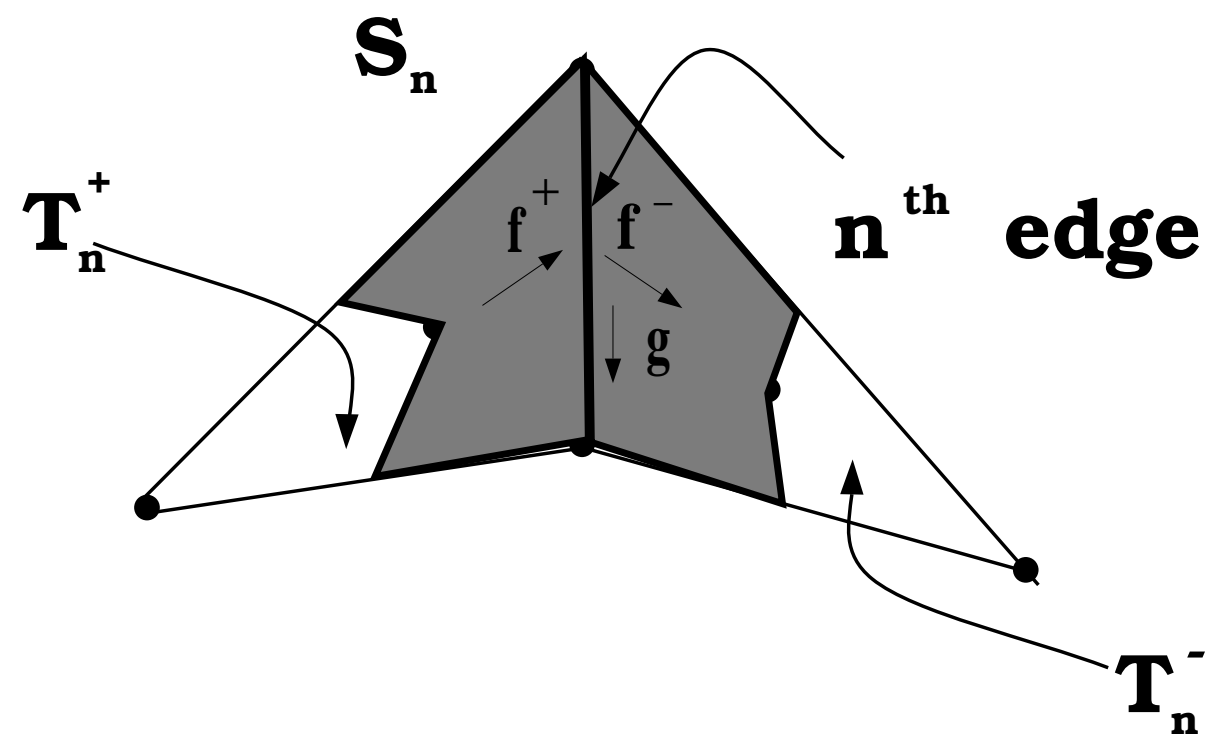

Figure 2: Basis function description: For the $n^{\text {th }}$ edge, basis functions $\boldsymbol{f}$ and $\boldsymbol{g}$ are defined in the shaded area. 


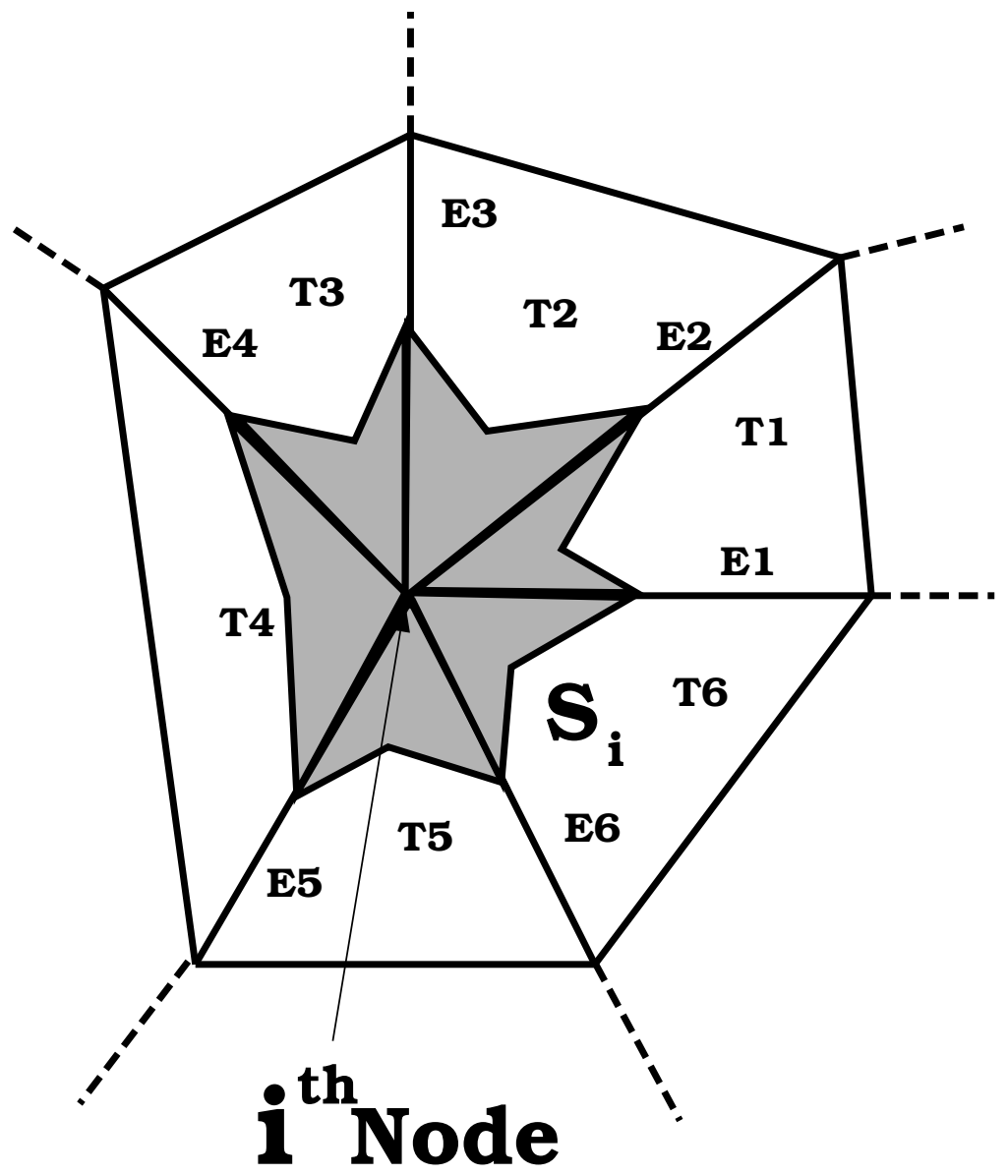

Figure 3: Charge patch around $i^{\text {th }}$ node. 


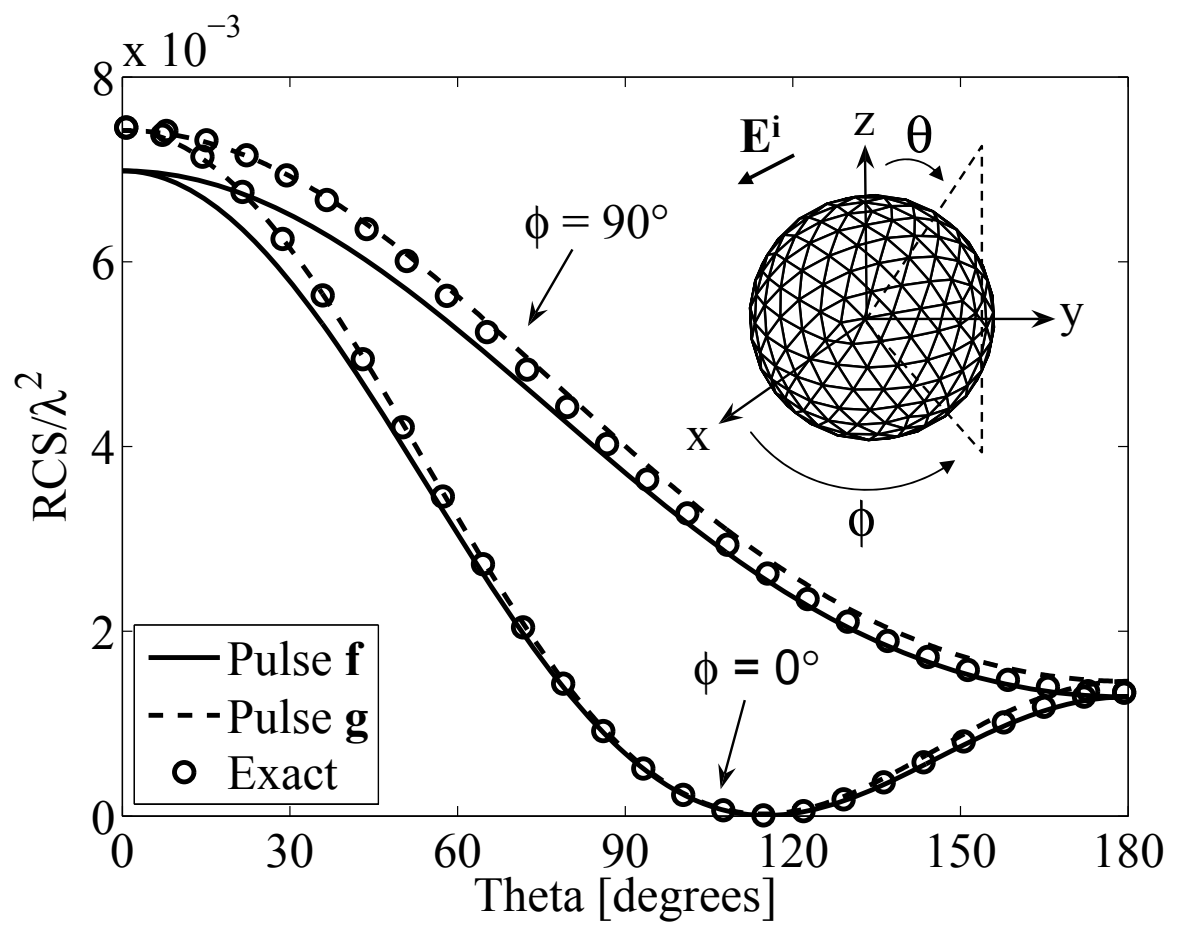

Figure 4: Bistatic RCS of a sphere of diameter $0.15 \lambda$ excited by a plane wave travelling in the $-\mathrm{z}$ direction. 


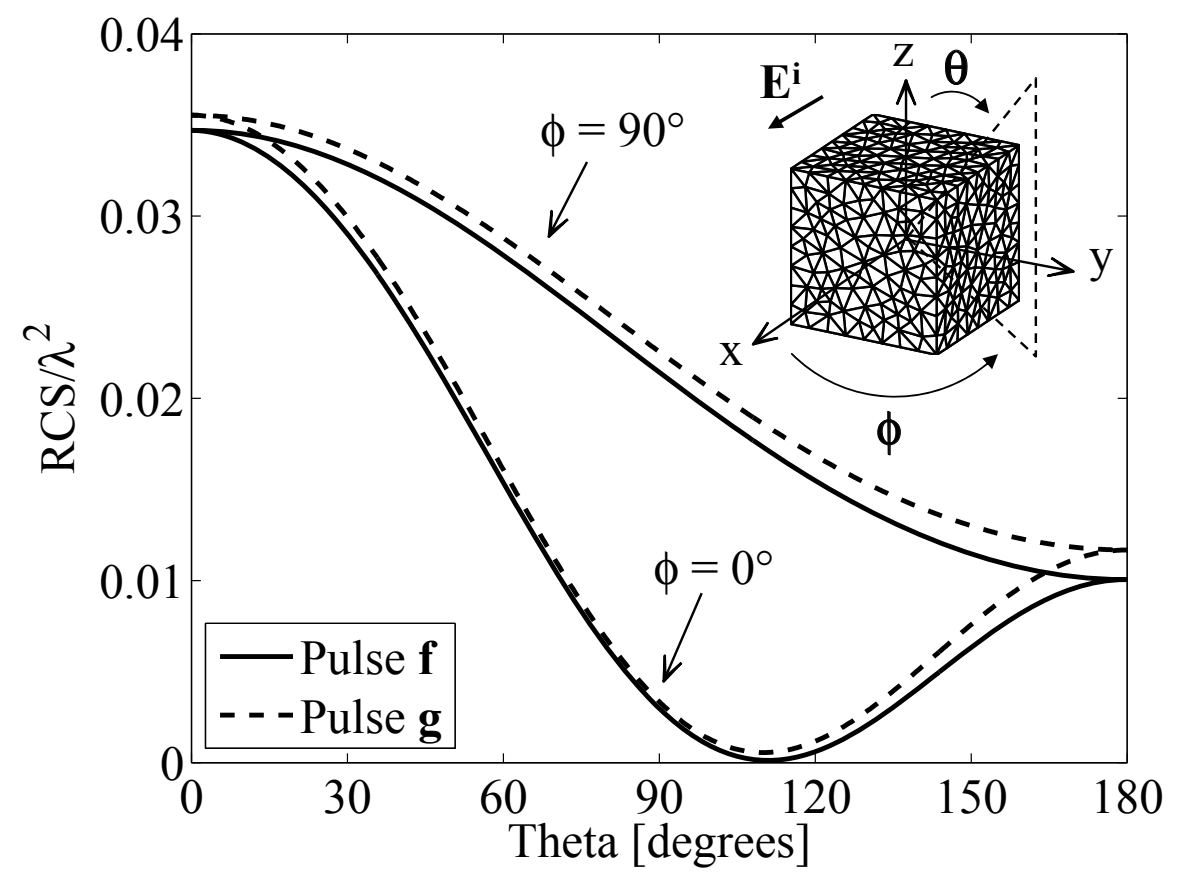

Figure 5: Bistatic RCS of a cube of length $0.15 \lambda$ excited by a plane wave travelling in the $-\mathrm{z}$ direction. 